\title{
Thermography for hyperthermia effects visualisation during magnetic stimulation in Image-Guided Radiation Therapy
}

\author{
by N. Ludwig, P. Arosio, S. Gallo, M. Gargano, J. Melada, and I. Veronese
}

\author{
* State University of Milano, Departement of Physics, via Celoria 1620133 Milano, Italy \\ Corresponding author: nicola.ludwig@unimi.it
}

\begin{abstract}
Gold fiducial markers, implanted into tumors, are commonly used during Image-Guided Radiation Therapy treatments for target localization and patient set up verification. In this study, hyperthermia effects produced by different types of millimetric gold fiducial markers under the application of an alternating magnetic field in the typical instrumental conditions used in Magnetic Fluid Hyperthermia clinical trials were investigated. Temperature monitoring of the gold fiducial markers into hydrogel-based tissue mimicking phantoms showed temperatures up to $70^{\circ} \mathrm{C}$ within tens of seconds and local increases to values higher than $45^{\circ} \mathrm{C}-50^{\circ} \mathrm{C}$ inside the hydrogel volume around fiducials, values able to induce thermal ablation of biological systems.
\end{abstract}

\section{Introduction}

Current treatment strategies for several oncological pathologies are based on the synergic combination of different techniques. Modern Radiation therapy (RT) increasingly uses conformal delivery techniques, which require an accurate patient positioning and tumor targeting. This aspect is even more important in the case of irradiation of moving organs, e.g. organs influenced by breathing. The most used imaging techniques in the so-called Image Guided Radiation Therapy (IGRT) are those based on X-rays. In such case, millimetric radiopaque metallic fiducial markers can be implanted into the lesions, or in their proximity. These markers are easily visible by X-ray imaging and allow target localization and patient set up verification. Meanwhile, Magnetic Fluid Hyperthermia (MFH) in combination with RT is recently gaining importance in oncology. In MFH technique, magnetic nanomaterials (normally iron oxides nanoparticles) directly implanted in the tumour mass at high doses (approximately $50 \mathrm{mg} / \mathrm{cm} 3$ ), under the action of an externally applied alternating magnetic field (AMF), can generate a certain amount of local heat depending on the frequency and the amplitude of field. The success of MFH was recently demonstrated on glioblastoma multiform and prostate cancers in clinical trials, that certify a survival extension of patients had undergone the combined therapy. In the present work we investigate the hyperthermia effects produced by IGRT fiducial markers stimulated by the application of an AMF in experimental conditions similar to those used in MFH clinical practice, i.e. frequency $f=109.8 \mathrm{kHz}$ and AMF of $16.15 \mathrm{kA} / \mathrm{m}$. The magnetic stimulation was obtained by means of Magnetherm (nanoThericsTM) set-up that builds up an AMF with amplitude values up to $17 \mathrm{kA} / \mathrm{m}$, and a frequency range from 100 to $1000 \mathrm{kHz}$.

\section{Matherials and methods}

Gold fiducial markers, sphere, cylinders and coils, were placed, individually, inside the homogenous magnetic field region using a suitable polystyrene support. During the AMF stimulation a high resolution thermal camera (FLIR A65 FLIR Systems Inc., $640 \times 512$ pixel, $0.05 \mathrm{~K}$ thermal resolution, $25 \mathrm{~mm}$ Germanium lens) was used to monitor the temperature variation of the fiducials over the time. After the start of the AMF stimulation, the temperature increased of more than $70^{\circ} \mathrm{C}$ in less than 30 seconds, reaching a final stable value of approximately $94^{\circ} \mathrm{C}$. Similar trends were obtained with the cylinders, using the same experimental set-up. Results clearly demonstrated the possibility to induce the heating of the gold fiducial markers under a suitable magnetic stimulation. The temperatures reached by the gold markers proved to be higher than those required to produce thermal ablation of biological systems, i.e. above $45-50^{\circ} \mathrm{C}$. 11,12 Therefore, it is worth studying the heat transfer properties occurring into soft tissues surrounding the fiducial markers in case of MFH treatments. In order to model this scenario, hydrogel phantoms based on poly(vinyl-alcohol) (PVA) cross-linked with glutaraldehyde (GTA) were prepared. GTA-PVA gel is a well-known matrix able to mimic efficiently the physiological thermal properties of tissues.

\section{Results}

Gold fiducial markers were dispersed inside the hydrogel, at a depth of approximately $2 \mathrm{~mm}$ from the phantom surface. The position of the gold markers into the gels was chosen to test the possible influence of the geometrical arrangement. Two different configurations were considered. The first consisted in placing a single fiducial marker in the center of the hydrogel phantom. In this set-up the cylinder and the helical coil were inserted both vertically-oriented and 
horizontally-oriented with respect to the gel surface. The thermal camera images of the hydrogel phantoms surface containing different individual fiducial markers acquired 60 seconds after the start of the AMF stimulation are shown in Figure 1. The heating curves measured on the phantom surface overran area of few pixels are given in Figure 2.

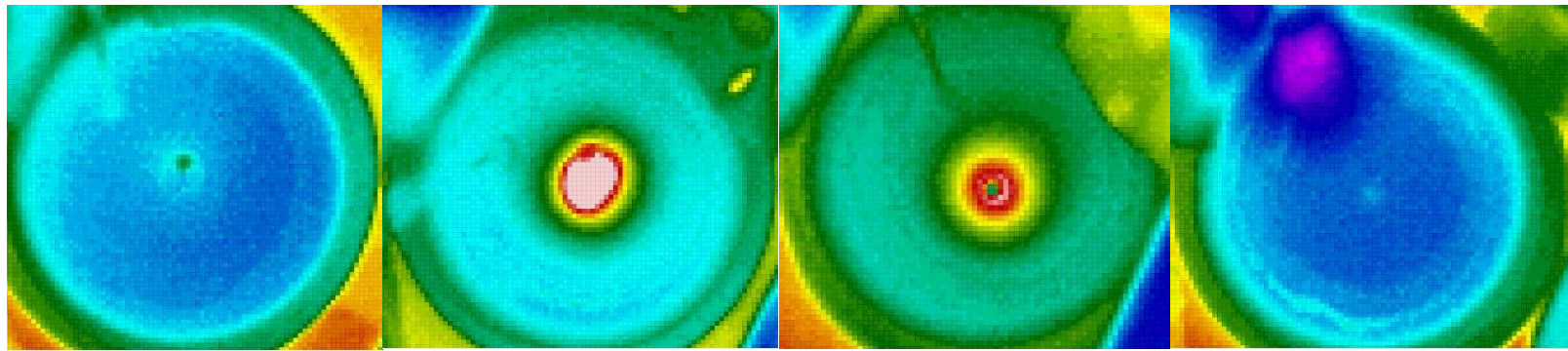

FIG. 1. Thermal images of hydrogel surface with Fiducial Markers (sphere) at different times after the start of the AMF stimulation: a) rod $\mathrm{V}, \mathrm{b}$ ) rod $\mathrm{H}$, c) sphere, d) coil, the apparent colder spot at the center of heated area in c) is an effect of the low emissivity of the surface of gold.

The thermal images revealed the progressive diffusion of the heat over the hydrogel surface, as effect of the increase of the temperature of the gold fiducial marker. The extent of such diffusion measured by the thermal camera depended on the type of fiducial marker and on the geometric configuration used. In particular, the diffusion was negligible in the case of the PointCoil ${ }^{\mathrm{TM}}$ Fiducial Marker (Fig. 1d) regardless of the vertical or horizontal orientation.

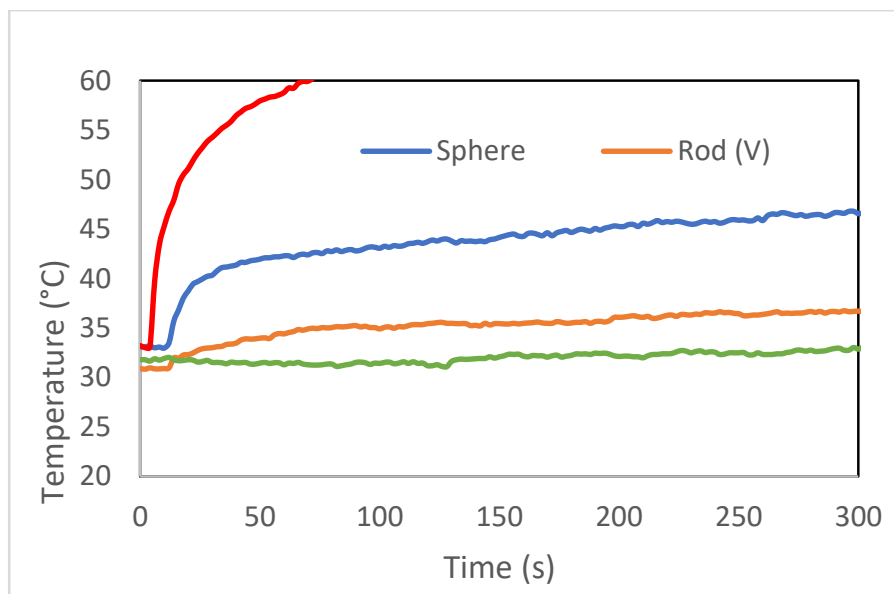

Fig. 2. Temperature profiles at different times, These profiles were obtained considering the hydrogel only avoiding the apparent cold spot of the fiducial marker due to the low emissivity of gold surface.

Results attested the increase of the hydrogel temperature due to the fiducial heating employed in IGRT when stimulated by a suitable AMF. Temperature increases were evaluated in the typical instrumental conditions used at present in MFH clinical trials and it makes sense to assume that the heat release by a fiducial marker in in-vivo condition should be higher. In our set up the upper side of the hydrogel is in air implying a different heat exchange with respect to the "in body" situation, where the markers are surrounded by tissues at $37^{\circ} \mathrm{C}$. The experimental evidences of this study highlight the need to evaluate carefully the location of fiducial markers in case of combined MFH/IGRT treatments, in order to avoid pain and detriment for patients. When fiducial markers are implanted into proximity of tumor an excessive heating might produce thermal ablation and damages of the health tissues surrounding the markers in a not negligible volume. This study suggests that the use of AMF stimulation could represent a further means to produce hyperthermia effects using millimetric gold structures once properly implanted into tumors.

\section{REFERENCES}

[1] Gallo, S., Arosio, P., Avolio, M., Bonizzoni, L., Cobianchi, M., Gargano, M., ... \& Veronese, I. (2018). 261. Hyperthermia properties of gold fiducial markers. Physica Medica, 56, 222-223.

[2] Arosio, P., Avolio, M., Gargano, M., Orsini, F., Gallo, S., Melada, J., ... \& Veronese, I. (2020). Magnetic stimulation of gold fiducial markers used in Image-Guided Radiation Therapy: Evidences of hyperthermia effects. Measurement, 151, 107242 